\title{
ALGUNOS ASPECTOS CRÍTICOS EN LA APLICACIÓN DEL IMPUESTO DE SOCIEDADES A LAS COOPERATIVAS SEGÚN EL RÉGIMEN FISCAL DE COOPERATIVAS. LA NECESIDAD DE SU REFORMA ${ }^{1}$
}

\author{
POR \\ Agustín ROMERO CIVERA ${ }^{2}$ y \\ Sergio MARÍ VIDAL ${ }^{3}$
}

\section{RESUMEN}

El presente trabajo pretende poner de manifiesto las dificultades que presenta la aplicación del régimen fiscal de cooperativas como consecuencia de algunas cuestiones específicas de este tipo societario como son: la existencia de distintos tipos de resultados que además tributan a tipos impositivos distintos; la dotación a unos fondos sociales obligatorios, deducibles, en todo o en parte, cuyo cálculo se puede realizar antes de impuestos o después de impuestos; las discrepancias existentes en la calificación dada a distintas partidas por la ley sustantiva y la ley fiscal. En todos los casos surgen problemas de aplicación práctica de difícil e incluso, en ocasiones, imposible solución para atender tanto a la legislación sustantiva como a la fiscal. Si además unimos la existencia de diferencias temporarias que pueden tener origen diverso, la imputación de las mismas a un resultado u otro, o incluso el seguimiento de su reversión se complica sobre manera.

Esta situación supone un clarísimo agravio comparativo frente a otras formas jurídicas, que no presentan, ni de lejos, semejante complejidad. Así pues, se proponen en el trabajo algunas soluciones, como serían la existencia de un único resultado con un único tipo de gravamen; o la tributación exclusiva de los resultados extracooperativos quedando los

\footnotetext{
${ }^{1}$ Este trabajo es parte del Proyecto de investigación "Información contable, financiación, resistencia a la crisis y cooperativas", financiado por el Programa de Apoyo a la Investigación y Desarrollo (PAID-06-12) de la Universitat Politècnica de València.

${ }^{2}$ Profesor Contratado Doctor. Centro de Investigación en Gestión de Empresas (CEGEA). Universitat Politècnica de València. Dirección de correo electrónico: aromero@ cegea.upv.es.

${ }^{3}$ Profesor Titular de Universidad. Centro de Investigación en Gestión de Empresas (CEGEA). Universitat Politècnica de València. Dirección de correo electrónico: smari@ cegea.upv.es.

REVESCO No 118 - Segundo Cuatrimestre 2015 - ISSN: 1885-8031 - www.ucm.es/info/revesco

http://dx.doi.org/10.5209/rev_REVE.2015.v118.48709
}

Fecha de recepción: 22/07/2014

Fecha de aceptación: 06/03/2015 
cooperativos exentos del impuesto sobre sociedades, al tributar posteriormente estos rendimientos en el impuesto sobre la renta de los socios de la cooperativa; o caso de mantenerse las dos bases con los dos tipos, eliminar las discrepancias entre las normas sustantivas y la ley fiscal, así como permitir imputar cualquier ajuste únicamente a la base imponible cooperativa.

Palabras clave: Fiscalidad de cooperativas, Impuesto de sociedades, Ley 20/1990, obsolescencia régimen fiscal de cooperativas, reforma régimen fiscal de cooperativas.

Claves Econlit: P13; K34; H22.

\title{
SOME CRITICAL ASPECTS IN THE APPLICATION OF THE CORPORATE TAX TO COOPERATIVES ACCORDING TO THE TAX REGIME OF COOPERATIVES. ITS NECESSARY REFORM
}

\begin{abstract}
This paper aims to highlight the difficulties that cooperatives have in the calculation of corporate tax as a consequence of the application of the tax regime of cooperatives. There are some specific questions which affect corporate tax calculation: a) the existence of different types of results, affected by different tax rates; b) the endowment to some obligatory social funds, totally or partially deductible, the calculation of which can be made before or after taxes; c) the discrepancies in the qualification of different items by the substantive law and the tax law imply some problems in practical application or, sometimes, impossible solutions to comply with both substantive legislation and tax legislation.

This situation implies a clear disadvantage when compared with other types of societies which are far less complex. As a result, we propose some solutions, such as: a) the existence of only one type of result, with only one tax rate; b) the exclusive taxation of the non-cooperative profit, resulting in tax exemption on cooperative profit, given that partners of the cooperative will pay it in their income tax later; c) maintaining both types of results with both tax rates but eliminating the discrepancies between the substantive law and the tax law, as well as allowing adjustments only to the cooperative result.
\end{abstract}

Keywords: Tax system of cooperatives, Corporate tax, Law 20/1990, obsolescence of tax regime of cooperatives, reform of tax regime of cooperatives. 


\section{INTRODUCCIÓN}

La Ley 20/1990 de régimen fiscal de cooperativas, remite continuamente a la legislación sustantiva (mercantil) para tipificar e incluso "homologar" ciertos requisitos o características de las cooperativas con impacto en materia fiscal (Alguacil Marí y Romero Civera, 2008). Así pues, no existen criterios propios para asegurar la uniformidad de trato fiscal y el cumplimiento del principio de igualdad, sino que se limita, en general, a «convalidar» el régimen sustantivo.

Y esta circunstancia, además supone un incremento de las diferencias de trato, pues la regulación establecida por las diferentes Comunidades Autónomas resulta muy variada (Alguacil Marí y Romero Civera, 2013), como, por ejemplo, en las dotaciones a los fondos sociales obligatorios, que recordemos tendrán impacto fiscal al ser deducibles (en todo o en parte) en el impuesto sobre sociedades.

El artículo 13 de la Ley 20/1990, en general, al regular las causas de pérdida de la condición de cooperativa protegida, remite a la legislación sustantiva. No obstante, esta homologación causa turbulencias con dicho régimen mercantil, pues en ocasiones realiza su propia versión de las características que debe tener la cooperativa. De este modo se producen, no sólo desigualdades de trato, sino incoherencias dentro del propio texto legal en relación con otros aspectos del régimen fiscal y que pondremos de manifiesto más adelante.

En cambio, en los artículos 8 y ss. de dicha Ley, cuando se enumeran aquellos requisitos necesarios para la especial protección, la ley los establece de forma autónoma (Alguacil Marí y Romero Civera, 2008). Estos requisitos están parcialmente inspirados en los que definían a las figuras cooperativas en la Ley General de Cooperativas 3/1987, vigente cuando se aprobó la Ley 20/1990, y por ello generan bastante confusión con los actualmente regulados en las leyes estatal y autonómicas en su aplicación concreta para cada una de las cooperativas afectadas. No cabe duda que la parálisis de la Ley 20/1990, frente a las sucesivas reformas de las leyes sustantivas de cooperativas no favorece la sincronía entre todas ellas.

Volviendo a las condiciones de pérdida de la protección, nos encontramos con otro aspecto problemático, cual es la separación entre resultados cooperativos y extracooperativos. Así, se señalan en el artículo 13.10 de la Ley 20/1990 las causas de pérdida de la protección. En el mismo se establecen tres mandatos: 1) No realizar más operaciones con terceros que las previstas en las leyes cooperativas; 2) No superar en ningún caso (aunque la normativa 
sustantiva lo permita) el límite del 50\% del total de operaciones; 3) cumplir las obligaciones establecidas en la normativa cooperativa respecto a que los rendimientos derivados de estas operaciones se contabilicen separadamente y se destinen al Fondo de reserva obligatorio.

El problema que plantea esta regulación es la definición de «operaciones cooperativizadas», concepto que no aparece explicado en la ley 20/1990, así como el de «terceros», por contraposición a «operaciones con socios», salvo en lo relativo a su inclusión en los rendimientos extracooperativos (art. 21 de la Ley 20/1990).

Por cuanto hace referencia a esta necesidad de contabilización separada, el art. 13 de la ley 20/1990 remite al cumplimiento de la obligación establecida en la normativa sustantiva, por lo que sólo si ésta lo contempla se incumpliría el requisito. En este sentido, tanto la ley estatal como algunas autonómicas reconocen la posibilidad de contabilización conjunta de resultados cooperativos y extracooperativos, aunque advierte de sus consecuencias fiscales. La Disposición Adicional sexta de la ley 27/1999 estableció que las cooperativas que no separaran resultados (incluso si su normativa lo permitía) perderían la condición de fiscalmente protegidas. También es de subrayar la excepción vasca, en la que no existe separación contable entre resultados cooperativos y extracooperativos.

Es esta separación de los distintos tipos de resultados un aspecto clave para la determinación del impuesto sobre sociedades en las cooperativas, al establecer la Ley 20/1990 la separación en dos bases imponibles, cooperativa y extracooperativa, a las que se aplicarán tipos de gravamen diferenciados.

Precisamente es esta cuestión la que genera problemas de aplicación práctica, al complicar sobremanera la determinación de la cuota a pagar por este impuesto por parte de las cooperativas. Algunas de estas dificultades son las siguientes:

a) ¿Qué pasa si la ley sustantiva califica un ingreso como cooperativo y la ley fiscal como extracooperativo? El caso más representativo son las plusvalías reinvertidas.

b) ¿Qué sucede si hay ajustes extracontables temporarios activos o pasivos? ¿Pueden ser imputables a esas dos bases imponibles a proporción, o son identificables en cada una?

c) ¿Qué ocurrirá en los años posteriores cuando reviertan esos ajustes temporarios?

d) ¿Qué pasará con la compensación de pérdidas cooperativas o extracooperativas los años subsiguientes a su reconocimiento? Puede ser polémico, porque dependerá del origen de las mismas. 
e) ¿Qué sucede con las deducciones en la cuota si son producto de hechos que se asignan en las diferentes bases imponibles?

Con todo ello, la mayor dificultad consiste en tener que calcular la cuota líquida cooperativa y la extracooperativa, con un sistema de ecuaciones, en el que, con la actual legislación, todas las variables interactúan. Aunque las hojas de cálculo permiten iterar, pretendemos proponer un sistema de ecuaciones con el que de forma directa podamos calcular las cuotas.

El cálculo de ambas cuotas líquidas, a pesar de que la Ley 20/1990 establezca una sola al haber obtenido ya una única cuota íntegra, resulta necesario para calcular las dotaciones a los Fondos obligatorios y estatutarios y para poder realizar correctamente el registro contable del impuesto, así como la regularización de las cuentas donde se recoge el impuesto devengado en el proceso de cierre contable. Pensemos, por ejemplo, que cuando las dotaciones a los fondos se realicen después de impuestos, tendremos que separar la parte del impuesto que se detrae del resultado cooperativo y del extracooperativo para poder determinar las cuantías sobre la que aplicar los porcentajes de dotación de los mismos. Por tanto, la cuota líquida deberá estar desglosada en la derivada del resultado cooperativo y la derivada del extracooperativo, lo que nos permitirá determinar separadamente la cuota que deducimos del resultado cooperativo y la que deducimos del extracooperativo. Del mismo modo, para el adecuado registro contable, necesitaremos determinar cuál es el gasto por impuesto sobre sociedades que imputaremos a las distintas cuentas que recogen el beneficio cooperativo (por ejemplo, “1291. Resultado cooperativo") y extracooperativo (por ejemplo, en la cuenta "1292. Resultado extracooperativo").

Por lo tanto, para poder conseguir lo que acabamos de señalar, incluso las deducciones deberán estar individualizadas en los dos tipos de cuota.

Intentaremos a continuación dar solución a estas cuestiones. Asimismo, recordaremos también la problemática ya tratada por otros autores (Caparrós Navarro, 1991; Romero Civera, 1992; Artacho Ruiz, Ceular Villamandos y Fuentes García, 2001) relacionada con la dotación a los fondos obligatorios, antes o después del cálculo del impuesto.

Como veremos, algunas de estas soluciones presentarán un grado de dificultad no pequeño, y en todo caso, sin parangón en el ordenamiento jurídico español. 
Asimismo, propondremos algunas alternativas, si bien supondrían una revisión profunda de la Ley 20/1990 citada.

\section{MÉTODOLOGÍA}

La metodología empleada en este trabajo combina la revisión de la normativa reguladora de las cooperativas, tanto sustantiva (leyes de cooperativas estatal y autonómicas actualmente vigentes) como fiscal (Ley 20/1990 de Régimen fiscal de cooperativas), con la aplicación de las mismas para el cálculo del impuesto sobre sociedades.

Para ello, contaremos con las 16 leyes de cooperativas autonómicas y la ley estatal, así como la Ley de régimen fiscal de cooperativas.

Revisaremos el contenido de estas normativas, de cara a su aplicación para el cálculo del impuesto sobre sociedades, poniendo de manifiesto las dificultades prácticas que surgen como consecuencia, en algunos casos del diferente criterio utilizado por éstas, y en otros por lo que entendemos como obsolescencia de una norma fiscal creada hace 24 años y que requiere una profunda revisión para su adaptación a la realidad actual.

\section{RESULTADOS}

\subsection{Determinación de los resultados según la ley 20/1990 de régimen fiscal de cooperativas}

La sección $2^{\text {a }}$ del capítulo IV del título II de la Ley 20/1990, establece la forma de determinar los resultados cooperativos, estableciendo los que se consideran ingresos y gastos cooperativos.

Por otro lado, la sección $3^{\mathrm{a}}$ de este mismo capítulo determina los resultados extracooperativos, estableciendo también los que se consideran ingresos extracooperativos y gastos extracooperativos.

La deuda tributaria será la suma algebraica de las cantidades resultantes de aplicar a las dos Bases Imponibles, cooperativa y extracooperativa, resulten positivas o negativas, los tipos de gravamen correspondientes. Si la suma algebraica resulta positiva tendrá la consideración de cuota íntegra. 
Si la cuota anterior resultara negativa, las cooperativas podrán deducirse la misma, en ejercicios posteriores, con las cuotas íntegras positivas de los cinco ejercicios siguientes (actualmente, en los diecisiete siguientes, como el resto de Sociedades hacen con sus bases imponibles negativas). Debe observarse que las cooperativas especialmente protegidas compensarán las cuotas, antes de aplicar su bonificación del 50\%, por lo que las cuotas pendientes de compensar nunca se entienden "bonificadas".

Los tipos de gravamen actuales son:

- El 20\% para resultados cooperativos en general, excepto las cooperativas de crédito.

- El 25\% para resultados cooperativos de cooperativas de crédito (fueron del $26 \%$ en la Ley original).

- El $30 \%$ para resultados extracooperativos y extraordinarios (fueron el 35\% en su versión original)

Llegados a este punto, es de subrayar que la adaptación del Plan General Contable a cooperativas (Orden EHA 3360/2010), considera la dotación al FEP como gasto, por lo que a efectos de la Base Imponible cooperativa el gasto contabilizado por la dotación al FEP es un gasto deducible si está por debajo del límite del 30\%, sin necesitar ningún ajuste en la Base Imponible.

Por el contrario, el 50\% de la dotación al FRO, necesitará un ajuste negativo tanto en la Base Imponible cooperativa como en la extracooperativa, excepto en el caso de la dotación obligatoria al FRO, cuando éste se haya clasificado como pasivo financiero (como consecuencia de ser considerado, al menos en parte, como repartible), puesto que la norma contable también lo registra como gasto contable en ese caso (Norma cuarta de la Orden EHA/3360/2010). Cuestión distinta será si debiera revisarse la pertinencia de seguir dando ese tratamiento fiscal a la dotación del FRO, en el caso de que este fuese parcial o totalmente repartible. ¿Qué sentido tiene que se considere deducible el 50\% de la dotación al FRO si este es repartible? Barberena Belzunce, 1992 y De Luís Esteban, 1996 ya opinaban en ese sentido, pero nada se contempla en la norma fiscal al respecto, tal vez porque en el momento de su elaboración ninguna ley de cooperativas reconocía la repartibilidad (aunque fuera parcial) del FRO, y posteriormente no ha sido objeto de modificación. No obstante, conviene señalar que no es práctica habitual en las cooperativas, al menos por el momento, reconocer en sus 
estatutos esta repartibilidad parcial, a pesar de que la Ley de cooperativas que le sea de aplicación sí la contemple.

Vemos como todos estos antecedentes descritos hacen que puedan surgir varios conflictos entre la contabilidad y la fiscalidad de las cooperativas, que conducen a incidencias importantes que complican el cálculo del impuesto sobre sociedades. Y es lo que vamos a analizar en los siguientes apartados.

\subsection{La dotación a fondos: antes o después del cálculo del impuesto}

Existen leyes sustantivas en las que la dotación a los Fondos obligatorios, FEP o FRO, se hace antes del cálculo del impuesto sobre Sociedades y otras, en que se hace después (tabla 1). No obstante, la situación legislativa ha ido cambiando en estos últimos años, a raíz de la adaptación contable de las cooperativas al Plan General de Contabilidad de 2007, por el que las leyes sustantivas incluyeron modificaciones en la parte económica y, sobre todo, en la discutida y discutible calificación del capital social como patrimonio neto o pasivo (Gómez Aparicio y Miranda García, 2006; Marí Vidal y Polo Garrido, 2012a, 2012b).

Tabla 1. La dotación a los fondos sociales obligatorios antes o después de impuestos en las leyes de cooperativas actualmente vigentes.

\begin{tabular}{|l|c|c|}
\hline CCAA & $\begin{array}{c}\text { Dotación a inicio } \\
\text { de 2011 }\end{array}$ & $\begin{array}{c}\text { Dotación a inicio } \\
\text { de 2014 }\end{array}$ \\
\hline Andalucía & Después & Antes \\
\hline Aragón & Después & Antes \\
\hline Asturias & Antes & Antes \\
\hline Baleares & Antes & Antes \\
\hline Cantabria & No existía Ley & Antes \\
\hline Castilla la mancha & Antes & Antes \\
\hline Castilla y León & Antes & Antes \\
\hline Cataluña & Antes & Antes \\
\hline Euskadi & Después & Después \\
\hline Extremadura & Después & Después \\
\hline Galicia & Después & Antes \\
\hline La rioja & Antes & Antes \\
\hline Madrid & Después & No concreta \\
\hline
\end{tabular}




\begin{tabular}{|l|c|c|}
\hline Murcia & Antes & Antes \\
\hline Navarra & Después & No concreta \\
\hline C. Valenciana & Después & No concreta \\
\hline Ley estatal & Antes & Antes \\
\hline
\end{tabular}

Fuente: Elaboración propia a partir de ALGUACIL MARÍ y ROMERO-CIVERA, 2011

La casuística es diferente y puede llevar a algunas consecuencias ciertamente paradójicas o de cálculo más complicado:

- En el caso que se calcule el impuesto después de las dotaciones, puede resultar que, si los ajustes positivos a la base imponible son muy importantes, el impuesto sea tan grande que no haya suficiente beneficio para dotar los Fondos obligatorios (FEP o FRO) especificados en la Ley sustantiva o los Estatutos. Este hecho afectaría especialmente el FEP, que se considera en su totalidad como gasto contable antes del cierre (recordemos que según lo establecido en la Orden EHA 3360/2010, en aquellos casos en los que el FRO sea repartible, la dotación obligatoria también se considerará gasto contable). Por lo tanto, se haría imposible hacer las dotaciones tal como prevén las leyes sustantivas o los estatutos. Surgiría un "bucle", en el que necesariamente habría que priorizar con el impuesto efectivamente devengado, aunque no se cumpliera la Ley mercantilsustantiva. Es evidente que los legisladores que han decidido hacer la dotación antes del cálculo del Impuesto, no preveían estas circunstancias.

- Si se hace el cálculo de la dotación a Fondos (FEP y FRO) después del cálculo del impuesto, que es una acción más congruente para evitar el efecto anterior, éstas intervienen en el propio cálculo del impuesto al considerarse deducibles (en un 50\% en el caso del FRO), necesitando un sistema de ecuaciones u hojas de cálculo para resolverlo. Sin duda es una situación excepcional que no conocemos que se produzca en otros tipos societarios, al menos afectando a un número tan importante de empresas, como ocurre en el caso de las miles de cooperativas sujetas a esta mecánica.

Así, si eliminamos del resultado contable los gastos registrados por la dotación del FEP (y los del FRO cuando así se hubiesen registrado), considerando la deducción de los 
mismos como ajustes a la base imponible ${ }^{4}$, y suponiendo que no exista ningún ajuste a la base imponible que no sean los del FEP y el FRO, tendremos que:

1) Si el cálculo de las dotaciones a FEP y FRO es anterior al del Impuesto:

$\mathrm{XC}=0.2 *(\mathrm{BAIC}-\mathrm{a} * \mathrm{BAIC}-0.5 * \mathrm{~b} * \mathrm{BAIC})=0.2 * \mathrm{BAIC} *(1-\mathrm{a}-0.5 * \mathrm{~b})$

$\mathrm{XE}=0.3 *\left(\mathrm{BAIE}-0.5^{*} \mathrm{c} * \mathrm{BAIE}\right)=0.3 * \mathrm{BAIE}^{*}\left(1-0.5^{*} \mathrm{c}\right)$

2) Si las dotaciones a FEP y FRO son posteriores al cálculo del impuesto:

$\mathrm{XC}=0.2 *(\mathrm{BAIC}-\mathrm{a} *(\mathrm{BAIC}-\mathrm{XC})-0.5 * \mathrm{~b} *(\mathrm{BAIC}-\mathrm{XC}))$

$\mathrm{XE}=0.3 *\left(\mathrm{BAIE}-\left(0.5 * \mathrm{c}^{*}(\mathrm{BAIE}-\mathrm{XE})\right)\right.$

Que despejando, resultaría:

$\mathrm{XC}=\mathrm{BAIC} * 0.2 *(1-\mathrm{a}-0.5 * \mathrm{~b}) /(1-0.2 * \mathrm{a}-0.1 * \mathrm{~b})$

$\mathrm{XE}=\mathrm{BAIE} * 0.3 *(1-0.5 * \mathrm{c}) /\left(1-0.15^{*} \mathrm{c}\right)$

Donde:

$\mathrm{XC}=$ cuota del impuesto derivada del resultado cooperativo

$\mathrm{XE}=$ cuota del impuesto derivada del resultado extracooperativo

BAIC: Beneficio cooperativo antes de impuestos = Base Imponible de la base cooperativa (excluidos los gastos por la dotación obligatoria del FRO y FEP)

BAIE: Beneficio extracooperativo antes de impuestos = Base Imponible de la base extracooperativa (excluidos los gastos por la dotación obligatoria del FRO)

a= coeficiente de dotación estatutario al FEP (máximo 30\%)

$\mathrm{b}=$ coeficiente de dotación estatutario al FRO de los resultados cooperativos

$\mathrm{c}=$ coeficiente de dotación estatutario al FRO de los resultados extracooperativos

Lo que nos hace ver el inicio de la dificultad de cálculo.

\subsection{Diferencias de criterio entre la normativa sustantiva y la fiscal}

Si hay diferencias de criterio entre lo que considere cooperativo o extracooperativo la ley sustantiva-mercantil que regula una cooperativa y la ley fiscal, surgen evidentes problemas de cálculo. El ejemplo más típico son los resultados positivos por la venta de inmovilizado que se reinvierten: en todas las leyes sustantivas se consideran cooperativos y en la fiscal, extracooperativos.

\footnotetext{
${ }^{4}$ Caparrós Navarro ya exponía en 1991 la necesidad de operar con ecuaciones para resolver las dotaciones a los Fondos y las cuotas correspondientes
} 
Este hecho produce dos reajustes en las dos Bases Imponibles, con aumentos en la extracooperativa y disminuciones en la cooperativa. Es decir al resultado cooperativo le deberíamos restar ese beneficio para llevarlo a la Base imponible extracooperativa.

Pero además, este ajuste debe "arrastrar" también a la dotación al FEP, efectuada por ese beneficio reinvertido (frecuentemente por importe muy elevado), de la base cooperativa a la extracooperativa. Y lo mismo sucederá con la dotación al FRO correspondiente a ese beneficio reinvertido que acabamos de reclasificar que deberá restarse de la base extracooperativa y no de la cooperativa. Nada dice la Ley Fiscal al respecto, pero por pura coherencia, sería lógico considerarla como un ajuste negativo en la Base imponible extracooperativa y un ajuste positivo en el resultado cooperativo. En caso contrario, podríamos no tener suficiente base imponible cooperativa para imputar los ajustes negativos derivados de la deducibilidad de estas dotaciones.

Existen otros tipos de ingresos que tienen consideraciones clasificatorias divergentes entre las dos legislaciones, de los que destacamos los siguientes: parte de los ingresos procedentes de las secciones de crédito, y los dividendos derivados de una sociedad vinculada subordinada o complementaria. Estos casos también son considerados como ingresos cooperativos en las leyes sustantivas y extracooperativos en la fiscal. No hemos encontrado casos a la inversa.

Siguiendo con los dos tipos de ecuaciones, añadimos más parámetros relacionados:

$\mathrm{BAIR}=$ Beneficio antes de impuestos cooperativo reclasificado que tributa en la Base Imponible extracooperativa

$\mathrm{XR}=$ cuota de impuesto derivada del resultado que siendo cooperativo en la ley sustantiva, tributa como extracooperativo

1) Si se calculan las dotaciones antes del cálculo del impuesto:

$$
\begin{aligned}
& \mathrm{XC}=0.2 *((\mathrm{BAIC}-\mathrm{BAIR})-\mathrm{a} *(\mathrm{BAIC}-\mathrm{BAIR})-0.5 * \mathrm{~b} *(\mathrm{BAIC}-\mathrm{BAIR})) \\
& \mathrm{XE}=0.3 *(\mathrm{BAIE}-0.5 * \mathrm{c} * \mathrm{BAIE}) \\
& \mathrm{XR}=0.3 *(\mathrm{BAIR}-\mathrm{a} * \mathrm{BAIR}-0.5 * \mathrm{~b} * \mathrm{BAIR})
\end{aligned}
$$

Que despejando resultaría

$$
\begin{aligned}
& \mathrm{XC}=0.2 * \operatorname{BAIC} *(1-\mathrm{a}-0.5 * \mathrm{~b})-0.2 * \operatorname{BAIR} *(1-\mathrm{a}-0.5 * \mathrm{~b})=0.2 *(1-\mathrm{a}-0.5 * \mathrm{~b}) *(\text { BAIC-BAIR }) \\
& \mathrm{XE}=0.3 * \mathrm{BAIE}^{*}(1-0.5 * \mathrm{c}) \\
& \mathrm{XR}=0.3 * \mathrm{BAIR}^{*}(1-\mathrm{a}-0.5 * \mathrm{~b})
\end{aligned}
$$


2) Si se calculan las dotaciones tras el cálculo del impuesto:

$\mathrm{XC}=0.2 *((\mathrm{BAIC}-\mathrm{BAIR})-\mathrm{a} *((\mathrm{BAIC}-\mathrm{XC})-(\mathrm{BAIR}-\mathrm{XR}))-0.5 * \mathrm{~b} *((\mathrm{BAIC}-\mathrm{XC})-(\mathrm{BAIR}-\mathrm{XR})))$

$\mathrm{XE}=0.3 *\left(\mathrm{BAIE}-\left(0.5 * \mathrm{c}^{*}(\mathrm{BAIIE}-\mathrm{XE})\right)\right.$

$\mathrm{XR}=0.3 *(\mathrm{BAIR}-\mathrm{a} *(\mathrm{BAIR}-\mathrm{XR})-0.5 * \mathrm{~b} *(\mathrm{BAIR}-\mathrm{XR})$

Que despejando, tendríamos un sistema de ecuaciones:

$X C^{*}(1-0.2 * a-0.1 * b)=0.2 *(B A I C-B A I R) *(1-a-0.5 * b)+X R(-0.2 * a-0.1 * b)$

$\mathrm{XE}=\mathrm{BAIE}^{*} 0.3^{*}\left(1-0.5^{*} \mathrm{c}\right) /\left(1-0.15^{*} \mathrm{c}\right)$

$\mathrm{XR}=\operatorname{BAIR} * 0.3 *(1-\mathrm{a}-0.5 * \mathrm{~b}) /(1-0.3 * \mathrm{a}-0.15 * \mathrm{~b})$

\subsection{Ajustes extracontables temporarios que se asignan a alguna base imponible}

Si hay ajustes extracontables temporarios, pueden ser imputables a esas dos bases imponibles, pero los criterios pueden ser tanto directos como indirectos. Es decir, usar criterios de relación directa entre ingresos y gastos, o bien de forma proporcional. Por ello nos podemos encontrar ajustes a la base imponible:

- Cooperativa, cuando el ingreso o gasto afectado por el ajuste esté en esa Base. Por ejemplo, en la venta a un socio que resulta impagada y que presenta una antigüedad inferior a seis meses. Fiscalmente no sería deducible hasta que pasaran 6 meses, lo que genera un ajuste temporario positivo de activo.

- Extracooperativa, si en vez de afectarse en esa base lo fuera en la base extracooperativa. Por ejemplo, el mismo caso que el anterior, pero por la venta impagada por un "no socio" o "tercero", que evidentemente figura en los resultados extracooperativos.

- Proporcionalmente a las dos bases, cuando el ingreso o gasto no sea afecto a ninguna de las dos en particular. Por ejemplo, una provisión genérica sobre los saldos deudores.... Y sobre todo, los incentivos en amortizaciones (acelerada o libre) que conducen a ajustes negativos de pasivo los primeros años de utilización del elemento de inmovilizado. En este caso, aunque la Ley fiscal 20/90 no indique nada, la lógica conduciría a una imputación en la base proporcional. Pero ¿proporcional al volumen de operaciones total cooperativo o extracooperativo del año que se utilizan, según criterio sustantivo - mercantil? ¿o proporcional a las dos bases Imponibles, según criterio fiscal? Nos volvemos a encontrar ante una indecisión y un nuevo "bucle" de cálculo en que las variables están interrelacionadas.

No obstante, en este caso, la proporción por la que nos inclinamos, sería la que se base en un criterio sustantivo - mercantil porque, por un lado, el criterio de imputación de la 
Ley 20/1990 respeta los criterios que estén fundados en la legislación mercantil y por otro, y más importante, ése será el criterio que se habrá usado previamente para realizar el reparto contable del gasto o ingreso para la determinación de los resultados cooperativos o extracooperativos.

Por lo tanto añadimos nuevos parámetros:

$\mathrm{p}=$ ajustes permanentes en la base imponible cooperativa

$\mathrm{q}=$ ajustes permanentes en la base imponible extracooperativa

$\mathrm{r}=$ ajustes permanentes en los resultados cooperativos imputados fiscalmente como extracooperativos

$\mathrm{s}=$ ajustes temporarios en la base imponible cooperativa

$\mathrm{t}=$ ajustes temporarios en la base imponible extracooperativa

$\mathrm{u}=$ ajustes temporarios en la base imponible extracooperativa correspondientes a los resultados reimputados como extracooperativos

$\mathrm{z}=$ coeficiente (proporción sobre el total) de operaciones con terceros aplicado en la cooperativa basado en criterios contables fundados

$\mathrm{y}=$ proporción que representan en el total de operaciones, los resultados cooperativos reimputados como extracooperativos

$\mathrm{A}=$ ajustes temporarios a imputar al ejercicio de forma proporcional

$\mathrm{B}=$ ajustes permanentes a imputar al ejercicio de forma proporcional

Volviendo a los dos sistemas de cálculo, tendríamos

1) Si se calculan las dotaciones antes del cálculo del impuesto:

$\mathrm{XC}=0.2^{*}((\mathrm{BAIC}-\mathrm{BAIR})-\mathrm{a} *(\mathrm{BAIC}-\mathrm{BAIR})-0.5 * \mathrm{~b} *(\mathrm{BAIC}-\mathrm{BAIR})+\mathrm{p}+\mathrm{s}-\mathrm{r}-\mathrm{u}+(\mathrm{A}+\mathrm{B}) *(1-$ $\mathrm{z}-\mathrm{y})$ )

$$
\begin{aligned}
& \mathrm{XE}=0.3 *\left(\mathrm{BAII}-0.5^{*}(\mathrm{c} * \mathrm{BAIE})+\mathrm{q}+\mathrm{t}+(\mathrm{A}+\mathrm{B}) * \mathrm{z}\right) \\
& \mathrm{XR}=0.3 *\left(\mathrm{BAIR}-\mathrm{a} * \mathrm{BAIR}-0.5^{*} \mathrm{~b} * \mathrm{BAIR}+\mathrm{r}+\mathrm{u}+(\mathrm{A}+\mathrm{B}) * \mathrm{y}\right)
\end{aligned}
$$

Que despejando resultaría

$$
\begin{aligned}
& \mathrm{XC}=0.2 *(1-\mathrm{a}-0.5 * \mathrm{~b}) *(\mathrm{BAIC}-\mathrm{BAIR})-0.2 *(\mathrm{p}+\mathrm{s}-\mathrm{r}-\mathrm{u}+(\mathrm{A}+\mathrm{B}) *(1-\mathrm{z}-\mathrm{y})) \\
& \mathrm{XE}=0.3 * \mathrm{BAIE}^{*}(1-0.5 * \mathrm{c})+0.3 *(\mathrm{q}+\mathrm{t}+(\mathrm{A}+\mathrm{B}) * \mathrm{z}) \\
& \mathrm{XR}=0.3 * \mathrm{BAIR} *(1-\mathrm{a}-0.5 * \mathrm{~b})+0.3 *(\mathrm{r}+\mathrm{u}+(\mathrm{A}+\mathrm{B}) * \mathrm{y})
\end{aligned}
$$

2) Si se calculan las dotaciones tras el cálculo del impuesto: 


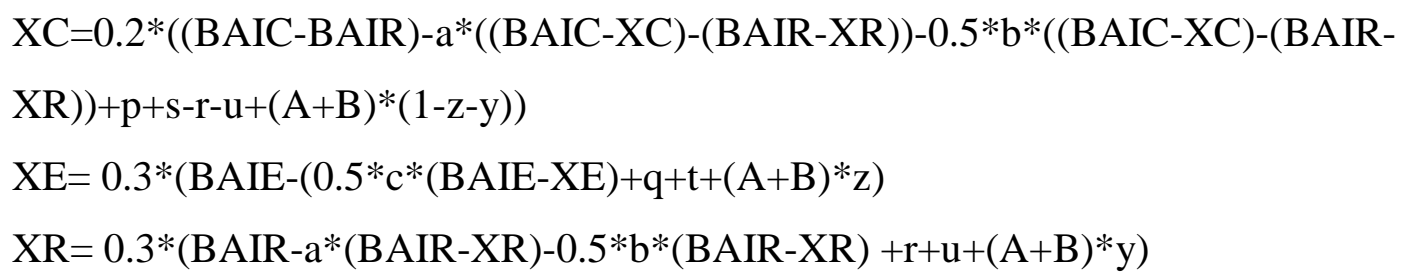

Que despejando, tendríamos un sistema de ecuaciones:

$$
\begin{aligned}
& \text { XC* }(1-0.2 * a-0.1 * b)=0.2 *(B A I C-B A I R) *(1-a-0.5 * b)+X R(-0.2 * a-0.1 * b)+ \\
& 0.2 *(\mathrm{p}+\mathrm{s}-\mathrm{r}-\mathrm{u}++(\mathrm{A}+\mathrm{B}) *(1-\mathrm{z}-\mathrm{y})) \\
& \mathrm{XE}=\left(\mathrm{BAIE}^{*} 0.3^{*}\left(1-0.5^{*} \mathrm{c}\right)+0.3 *(\mathrm{q}+\mathrm{t}+(\mathrm{A}+\mathrm{B}) * \mathrm{z}) /\left(1-0.15^{*} \mathrm{c}\right)\right. \\
& \mathrm{XR}=(\mathrm{BAIR} * 0.3 *(1-\mathrm{a}-0.5 * \mathrm{~b})+0.3 *(\mathrm{r}+\mathrm{u}+(\mathrm{A}+\mathrm{B}) * \mathrm{y}) /(1-0.3 * \mathrm{a}-0.15 * \mathrm{~b})
\end{aligned}
$$

\subsection{Los ajustes extracontables temporarios revierten}

El Plan General Contable establece que los Impuestos diferidos descritos en el punto anterior deben controlarse mediante dos cuentas contables, la "4740.- Activos por diferencias temporarias deducibles" y la “479.- Pasivos por diferencias temporarias imponibles".

Pero los importes de estas cuentas deben revertir, en el sentido de saldarse, cuando el criterio contable y fiscal difiera en sentido contrario al que difería inicialmente. Así por ejemplo, un incentivo a la amortización, permitirá amortizar fiscalmente antes que contablemente, pero al final, la amortización será del 100\% en los dos casos.

Estas cuentas se utilizan por la cuantía resultante de calcular cada ajuste en la base imponible multiplicado por el tipo de gravamen aplicado por la Sociedad. Pero dado que en el caso de las cooperativas, como acabamos de ver, hay dos bases con dos tipos de gravamen y los ajustes se han hecho incluso de modo proporcional, nos encontramos con los siguientes importes: $\mathrm{s}^{*} 0.2 ; \mathrm{A}^{*}(1-\mathrm{z}-\mathrm{y})^{*} 0.2 ; \mathrm{t}^{*} 0.3 ; \mathrm{u}^{*} 0.3 ; \mathrm{A}^{*} \mathrm{z}^{*} 0.3 ; \mathrm{A}^{*} \mathrm{y}^{*} 0.3$.

Como puede entenderse, todo ello hace extraordinariamente difícil el seguimiento contable de estas diferencias temporarias los años posteriores a través de las dos cuentas citadas. Especialmente complicado resulta para el caso de los ajustes proporcionales a las dos bases, ya que los criterios de proporcionalidad pueden, y suelen, ser diferentes cada año.

Tan difícil es, que en la práctica, los profesionales encargados de estos temas, optan por llevar todos los ajustes proporcionales sólo a la base cooperativa, que siempre suele ser más grande (porque las cooperativas tienen limitadas las operaciones con terceros hasta un 
máximo del 50\% o son descalificadas y tributarían en ese caso al régimen general de sociedades). Pero esa solución práctica, lejos de la exactitud, incumple las normas contables y evidentemente las fiscales, aunque para la inmensa mayoría de cooperativas con cuantías ínfimas. Pero obsérvese que este sería el resultado o consecuencia de la propia legislación "irracional" que las regulan.

Siguiendo nuestro esquema argumental, si en los años subsiguientes las proporciones de operaciones con terceros o de resultados reclasificados cambian, "z" ó "y" cambiarían, por lo que en las Bases imponibles respectivas, los ajustes necesarios para "revertir" los ajustes anteriores deberían ser controlados año por año, según el año en que se crearon.

Y ésta no es una dificultad teórica porque el porcentaje de operaciones con terceros en las cooperativas cambia efectivamente todos los años, ya que es prácticamente imposible que coincida, dada la evolución de los mercados, o la cifra de negocio, o la suma total de ingresos.

Aunque pudieran plantearse ecuaciones al respecto, llegamos a tal punto, que las ecuaciones serían enormes, y de muy difícil seguimiento e interpretación.

El control contable podría hacerse utilizando subcuentas específicas de la "479. Pasivos por diferencias temporarias imponibles" o "4740. Activos por diferencias temporarias deducibles" para diferenciar los ajustes que vienen de la base imponible cooperativa, de la extracooperativa o de la que surge tras la reclasificación de un resultado que siendo cooperativo en la ley sustantiva, tributa como extracooperativo, y que llamaremos reclasificada. Al año siguiente, cuando la proporción a imputar a un resultado u otro cambiara, deberíamos realizar ajustes a estos activos y/o pasivos por diferencias temporarias, de igual modo que se haría cuando cambiara por ejemplo el tipo de gravamen en una sociedad no cooperativa, utilizando para ello las cuentas "638. Ajustes positivos en la imposición sobre beneficios", o "633. Ajustes negativos en la imposición sobre beneficios". Pero año tras año, sean 1, 5, 10 ó 50 años deberíamos controlar y actualizar todos y cada uno de los ajustes de todos los años anteriores, afectados por los cambios de "z" o "y", además de los 3 directos, "s", "t", y "u", a los que no afectan las oscilaciones. Es evidente que tal propuesta, formalmente correcta, conduciría a una imposibilidad práctica de ejecución por lo que supone utilizar un número muy importante de variables durante un período de tiempo que puede ser de muchos años (tantos, por ejemplo, como el período de amortización del inmovilizado que 
originó la diferencia temporaria). En cualquier caso, esta dificultad resulta incomparable con otros tipos de sociedades.

\subsection{Existen pérdidas de ejercicios anteriores que se compensan}

Las pérdidas de ejercicios anteriores pueden tener un origen cooperativo, o extracooperativo, o mixto. En el caso de las cooperativas, compensan fiscalmente las cuotas negativas de ejercicios anteriores en vez de las bases, como el resto de sociedades, como consecuencia de tener el doble tipo impositivo (Marín Sánchez et al., 2012). Este cálculo se hace antes de bonificaciones y deducciones.

El control contable se efectúa a través de la cuenta "4745. Crédito por pérdidas a compensar del ejercicio", cuyo importe se calcula multiplicando el importe de la pérdida por el tipo de gravamen, siendo su saldo natural deudor. En la medida que se compensan se abonan hasta que se saldan.

Estas cuotas se restarían de la cuota íntegra, según la Ley 20/90, ya que aparentemente, al restar cuotas, ya no haría falta saber su origen. Pero el problema surge al tener que dar solución a la contabilización de la cuota líquida cooperativa o extracooperativa, tal y como ya se ha comentado en el apartado de introducción. Resulta necesario saber dónde debemos restar esas cuotas negativas, puesto que esta compensación influye en los cálculos. La iteración con hojas de cálculo sería una solución para poder determinar la cuantía máxima a compensar en cada tipo de cuota íntegra previa, hasta agotar el crédito impositivo en cada una de las tres cuotas. En caso de que la cuota total a compensar fuera superior a las tres cuotas previas del ejercicio actual, deberíamos también seleccionar cuál dejamos pendiente, ya que la ley no determina nada en concreto. También puede ocurrir que tengamos cuotas íntegras previas de distinto signo (por ejemplo, la cooperativa positiva y la extracooperativa negativa) en el mismo ejercicio. En este caso, de nuevo tendremos que acudir a cálculos iterativos para dar una solución adecuada.

Para estos casos más complicados deberíamos abandonar las ecuaciones $\mathrm{y}$, como se ha señalado, recurrir a hojas de cálculo con iteración. Por lo tanto el saldo de la cuenta "4745.Crédito por pérdidas a compensar del ejercicio" puede controlarse cronológicamente, según su origen para cálculos posteriores, aunque no siempre será necesario. No obstante, continua siendo posible un ajuste anual posterior en la medida que controlemos los parámetros " $\mathrm{z}$ " o 
"y" de años posteriores mediante subcuentas específicas a cada uno de los tres orígenes, directos o proporcionales, o sea 3 por 3 , un total de 9 subcuentas.

Finalmente, otra consideración que queremos resaltar, es la derivada de la disparidad de criterios a la hora de compensar pérdidas a partir de resultados futuros (tabla 2). La dificultad que podría entrañar esta forma de compensar las pérdidas, al poder tener que intervenir la dotación a los fondos obligatorios y el cálculo del impuesto sobre sociedades, con la complicación que este último tiene, como ya estamos poniendo de manifiesto en este trabajo, en la práctica no suele existir, puesto que la mayor parte de las veces las cooperativas optan por el saneamiento de esas pérdidas contra partidas de patrimonio neto, fundamentalmente reservas. No obstante, el crédito fiscal que se origina como consecuencia de la posibilidad de compensación de estas pérdidas con resultados futuros sí permanecerá en la contabilidad de la cooperativa en tanto no se compensen fiscalmente las mismas.

Tabla 2. Compensación de pérdidas a partir del resultado del ejercicio en las leyes de cooperativas actualmente vigentes

\begin{tabular}{|l|c|}
\hline CCAA & Compensación de pérdidas \\
\hline Andalucía & Antes de dotación y cálculo del IS \\
\hline Aragón & Después de dotación y cálculo del IS \\
\hline Asturias & Antes de dotación y cálculo del IS \\
\hline Baleares & Antes de dotación y cálculo del IS \\
\hline Cantabria & Antes de dotación y cálculo del IS \\
\hline Castilla la mancha & Antes de dotación y cálculo del IS \\
\hline Castilla y León & Antes de dotación y cálculo del IS \\
\hline Cataluña & Antes de dotación y cálculo del IS \\
\hline Euskadi & Antes de dotación y cálculo del IS \\
\hline Extremadura & Andes de la dotación y después del \\
\hline Galicia & cálculo del IS \\
\hline La rioja & Antes de dotación y cálculo del IS \\
\hline Madrid & Antes de dotación y cálculo del IS \\
\hline Murcia & Antes de dotación \\
\hline Navarra & Antes de dotación y cálculo del IS \\
\hline C. Valenciana & No son compensables \\
\hline
\end{tabular}




\begin{tabular}{|l|c|}
\hline & y las extracooperativas antes. \\
\hline Ley estatal & Antes de dotación y cálculo del IS \\
\hline
\end{tabular}

Fuente: Elaboración propia

\subsection{Existen deducciones con origen imputable a las dos bases imponibles}

La aplicación de las deducciones, aunque se efectúe sobre la cuota íntegra, también puede tener un origen, y una aplicación, en el cálculo de la cuota cooperativa o extracooperativa, o en la que hemos denominado reclasificada.

Piénsese que, por ejemplo, para saber el impuesto devengado por un resultado extracooperativo, la deducción por doble imposición debe estar presente en el cálculo. Por lo tanto, la deducción por un retorno cooperativo sería imputable al cálculo de la base imponible cooperativa y el impuesto devengado de tipo cooperativo, donde está el ingreso correspondiente. $\mathrm{Y}$ así mismo, la deducción por dividendo percibido de una sociedad participada sería imputable al extracooperativo porque en esa base está su imputación. Sin embargo, recuérdese que si la sociedad de la que se percibe el dividendo está subordinada a los fines de la cooperativa, dicho dividendo estará "reclasificado" como extracooperativo (la ley sustantiva lo clasifica como cooperativo).

No obstante, vuelve a reaparecer el caso de la imputación proporcional, para los casos en que la deducción sea "no asignable" a ninguno de los dos cálculos en concreto, como la deducción por I+D, o más difícil todavía, cuando la deducción sea por reinversión de beneficios extraordinarios, en la que como se expuso anteriormente, el origen está en un resultado cooperativo con ajustes del montante del beneficio y la dotación al FEP, que han ido a la base imponible extracooperativa. Al respecto, la consulta V1179-12, de fecha 31 de mayo de 2012, de la Dirección General de Tributos, advierte que no tiene deducción la parte del mismo que haya tenido la reducción en la base imponible correspondiente al 50\% del FRO. El control contable se efectúa a través de la cuenta de Activo "4742.- Derechos por deducciones y bonificaciones pendientes de aplicar", que va registrando las deducciones pendientes de aplicar por estar limitada su aplicación por insuficiencia de cuota, pues nunca puede ser negativa o existe una proporción según la cuota íntegra ajustada.

En el caso que exista limitación a la aplicación de deducciones sobre la cuota íntegra o la cuota íntegra ajustada, para las cooperativas surge un último dilema: si los cálculos mediante ecuaciones se han hecho linealmente, en el sentido de cuota cooperativa, 
extracooperativa o reclasificada ¿Cómo se aplica el límite si para una está excedido y en otro caso sobra? No habría más remedio de utilizar ya hojas de cálculo con iteración.

Con la casuística descrita, puede resultar inexplicable ya cualquier saldo contable de ajuste temporario.

\subsection{Coordinación de toda la problemática anterior}

Aunque aparentemente no tenga mayor dificultad para un experto en materia contable y fiscal, debe recordarse que, como existen dos bases imponibles con un tipo impositivo cada una, en la medida que exista interrelación en los ajustes antes descritos, por ser mixtos o por diferencia de criterio o por cuotas a compensar de origen mixto, la dificultad puede ser todavía mayor de la descrita. Las hojas de cálculo ayudan en gran manera a solucionar estos problemas, pero cuando se interrelacionan todos, la posibilidad de error es muy importante, además de una muy laboriosa preparación.

Y sobre todo, se trata de un agravio comparativo. Ningún otro tipo societario tiene estas dificultades de cálculo. Y no sólo se trata de las grandes cooperativas, pues hasta la más pequeña, en cuanto a volumen de operaciones, o número de socios, puede verse afectada.

Es decir, a la vista de todo lo anterior podrá entenderse la dificultad de muchas cooperativas en el cálculo del impuesto, con ecuaciones u hojas de cálculo complicadísimas, además de la exigencia de un potente historial de incidencias anteriores en los ajustes temporarios, que otros tipos de sociedades, ni de lejos, necesitan.

\section{CONCLUSIONES}

Con todo lo descrito, hemos pretendido demostrar la obsolescencia de la Ley 20/90 en lo que concierne al IS, al menos parcialmente ${ }^{5}$, y denunciar las complicaciones contables y fiscales que la existencia de dos bases y dos tipos tienen para las cooperativas, algo inaudito en la legislación cooperativa europea.

Ningún país significativo de nuestro entorno tiene para sus cooperativas dos bases imponibles ni dos tipos de gravamen: ni Francia, Italia, Alemania, Inglaterra, etc., ni tampoco

\footnotetext{
${ }^{5}$ De Luis Esteban, que fue uno de los artífices de la Ley 20/90, en 1996 ya admitía ciertas dificultades de aplicación práctica de la Ley, aún defendiendo su validez.
} 
aquellos con gran tradición cooperativa: Dinamarca, Holanda, Austria, etc.... (Montolio Hernández, 2000; Philipowsky, 1994; Münckner, 2008; Romero Civera, 2010).

Tan solo Portugal tenía un sistema similar, pero obsérvese la fecha de su creación: "Estatuto Fiscal Cooperativo Lei no 85/98 de 16-12-98, art ${ }^{\circ}$ " que evidentemente imitó la ley española.

A nuestro juicio, son varias las medidas que deberían implementarse para conseguir una norma fiscal más compatible con una aplicación práctica razonable, y que converja hacia los sistemas tributarios aplicados en los países de nuestro entorno. A continuación expondremos estas medidas, así como la justificación de las mismas.

A.- La existencia de un tipo de gravamen único ${ }^{6}$ es la más factible:

- Porque elimina toda la casuística de la complicación de cálculo, reasignación entre los dos tipos de bases, compensación de cuotas, etc.

- Porque, aunque la ley sustantiva prevea dos tipos de resultados, lo hace a efectos de las dotaciones a Fondos, por la diferencia de utilización de los resultados extracooperativos, que suele llevarlos hacia Fondos irrepartibles, a diferencia de los resultados cooperativos con más libertad de asignación: Retornos o reservas voluntarias.

- Porque, desde 1990, el tipo de gravamen se ha mantenido al $20 \%$ para la base cooperativa y sólo ha bajado del 35 al 30\% la extracooperativa, como las grandes empresas, cuando el resto de sociedades han tenido reducciones importantes: ERD, microempresas, empresas de nueva creación, etc. que han ido bajando, al 25, al 20 y al $15 \%$.

- Sería factible un tipo único entre el de microempresa y gran empresa o dependiendo de su tamaño o volumen. En caso de cambio de tipo, por esos motivos, los ajustes están previstos en el PGC: se efectuarían reajustando las diferencias temporarias originarias por los nuevos tipos impositivos y llevando a las cuentas "638. Ajustes positivos en la imposición sobre beneficios" y "633. Ajustes negativos en la imposición sobre beneficios", según el efecto que tuvieran en la cuenta de resultados. ... . De este modo lo ha resuelto la legislación foral vasca.

\footnotetext{
${ }^{6}$ La CCAE (Confederación de Cooperativas Agrarias de España) ahora "Cooperativas Agroalimentarias", efectuó una encuesta en 2011 entre sus asociadas, cuyas conclusiones, entre otras, iban en ese sentido.
} 
B.- Otra alternativa, en vez de mantener estas complicaciones de cálculo, aunque sería realmente innovadora para las cooperativas españolas, consistiría en legislar como la mayoría de países de nuestro entorno ${ }^{7}$. La mayoría de países de Europa con gran implantación de cooperativas (Alemania, Francia, Inglaterra, Holanda, etc.) han optado por la tributación efectivamente nula en el IS de los resultados cooperativos: tributan los socios (en IRPF o IS), imputándoseles a los mismos el resultado y evitando la doble imposición. Y por los resultados extracooperativos, las cooperativas tributan al régimen general del IS (Montolio Hernández, 2000; Philipowsky, 1994; Münckner, 2008; Romero Civera, 2010). La forma de resolverlo sería sencilla: Es deducible cualquier operación con los socios, sin ajustes por valor de mercado. De este modo, si existe base imponible, tributa como cualquier sociedad. El control reside en que los resultados que no procedan de socios tributen efectivamente. Para las cooperativas sería un avance en simplificación muy importante, y dado que los ingresos percibidos por los socios, al menos en los tipos de cooperativas más comunes (agrarias y trabajo asociado) ya se encuentran fiscalizados (por la retención sobre la liquidación realizada por la cooperativa a los socios en cooperativas agrarias, o la realizada en los anticipos laborales en cooperativas de trabajo asociado), estas rentas quedan perfectamente identificadas.

C.- La subsistencia de dos tipos de gravamen con dos bases imponibles debería mantenerse sólo en la medida en que todos los ajustes "mixtos" para el cálculo fueran "legalmente" asignables a la base cooperativa, de modo que no hubiera interferencias de la ley fiscal sobre la ley sustantiva. Es decir:

- Que no hubiera diferencias de criterio en la determinación de los resultados sobre la ley sustantiva, entre cooperativos y extracooperativos.

- Que todos los ajustes se hicieran sobre la base imponible cooperativa, salvo los que fueran totalmente de origen extracooperativo.

- Que una vez hecho el cálculo de las dos bases por sus tipos de gravamen, al haber obtenido una cuota previa, la compensación de cuotas se hiciera a discreción del sujeto pasivo en el cálculo, así como las deducciones.

\footnotetext{
${ }^{7}$ Alguacil Marí y Romero Civera, en diciembre de 2008, presentaron una propuesta de modificación de la Ley 20/90 en CEPES (Confederación Española de Empresas de la Economía Social) en ese sentido, aunque no prosperó.
} 
No obstante, vistos los cambios legislativos implicados, tanto de leyes de carácter cooperativo, mercantil y fiscal, nos parece, esta última, una propuesta de difícil aplicación.

En conclusión, es más coherente la primera propuesta, y ello supondría un drástico cambio en la Ley 20/90 en cuanto a la forma de cálculo del IS, pero no en los aspectos más "políticos" de la misma en cuanto a la protección fiscal, ya que podría, como en la actualidad, descalificarse a la cooperativa que no cumpla los requisitos expuestos en la misma, tributando como cualquier sociedad. Además, no sería necesario el cambio de las leyes sustantivas ni del propio PGC.

No obstante la segunda propuesta, "homologada" ya en Europa, simplificaría al máximo la gestión del impuesto, aunque conllevaría, seguramente un cascada de cambios normativos en las leyes sustantivas, como sucedió con la adaptación al PGC.

\section{BIBLIOGRAFÍA}

ALGUACIL MARÍ, P. y ROMERO-CIVERA, A. (2008) Criterios para una propuesta de régimen fiscal de cooperativas. Confederación Empresarial Española de la Economía Social, $73 \mathrm{p}$.

ALGUACIL MARÍ, P. y ROMERO-CIVERA, A. (2011) Requisitos para la aplicación del régimen fiscal especial de cooperativas. Quincena Fiscal Aranzadi, № 21/2011.

ALGUACIL MARÍ, P. y ROMERO-CIVERA, A. (2013) Diferencias territoriales en el concepto de cooperativa protegida y especialmente protegida. REVESCO. Revista de Estudios Cooperativos, № 110, pp. 7-42.

ARTACHO RUIZ, C.; CEULAR VILLAMANDOS, N. y FUENTES GARCÍA, F.J. (2001)

La problemática de cálculo del impuesto sobre sociedades en las cooperativas de ámbitos estatal y andaluz. Un caso práctico de análisis comparativo. CIRIEC-España, Revista de Economía Pública, Social y Cooperativa, No 38, pp. 155-170.

BARBERENA BELZUNCE, I. (1992) Sociedades cooperativas, anónimas laborales y agrarias de transformación. Régimen Fiscal. E. Aranzadi.

CAPARRÓS NAVARRO, A. (1991) Las sociedades cooperativas y el Impuesto de Sociedades: Armonización contable y fiscal. Revista de Contabilidad y Tributación, (Centro de Estudios Financieros), № 104, pp. 3-62. 
CONFEDERACIÓN DE COOPERATIVAS AGRARIAS DE ESPAÑA (2011) Informe Sobre Reforma Del Régimen Económico y Fiscal de las Cooperativas Agroalimentarias, 110 pp. Disponible en: http://www.agro-alimentarias.coop/ficheros/doc/02718.pdf.

DIRECCIÓN GENERAL DE TRIBUTOS (2012) Consulta vinculante n $^{\circ} 1179-12$, de 31 de mayo de 2012 de la Dirección General de Tributos.

DE LUÍS ESTEBAN, J.M. (1996) El Impuesto de Sociedades y la fiscalidad de las cooperativas: reforma y continuidad. CIRIEC-España, Revista de Economía Pública, Social y Cooperativa, $\mathrm{N}^{\circ} 23$, pp. 33-46.

GÓMEZ APARICIO, P. y MIRANDA GARCÍA, M. (2006) La caracterización financiera y contable del capital social a la luz de los principios cooperativos. REVESCO. Revista de Estudios Cooperativos, $\mathrm{N}^{\circ}$ 90, pp. 7-27.

LEY 1/2003, de 20 de marzo, de Cooperativas de las Illes Balears.

LEY 2/1998, de 26 de marzo, de Sociedades Cooperativas de Extremadura.

LEY 3/1987, de 2 de abril, General de Cooperativas (derogada).

LEY 4/1993, de 24 de junio, de Cooperativas de Euskadi.

LEY 4/1999, de 30 de marzo, de Cooperativas de la Comunidad de Madrid.

LEY 4/2001, de 19 de julio, de Cooperativas de La Rioja.

LEY 4/2002, de 11 de abril, de Cooperativas de la Comunidad de Castilla y León.

LEY 5/1998, de 18 de diciembre, de Cooperativas de Galicia.

LEY 8/2003, de 24 de marzo, de Cooperativas de la Comunidad Valenciana.

LEY 8/2006, de 16 de noviembre, de Sociedades Cooperativas de la Región de Murcia.

LEY 9/1998, de 22 de diciembre, de Cooperativas de Aragón.

LEY 11/2010, de 4 de noviembre, de Cooperativas de Castilla - La Mancha.

LEY 14/2011, de 23 de diciembre, de Sociedades Cooperativas Andaluzas.

LEY 14/2013, de 27 de septiembre, de apoyo a los emprendedores y su internacionalización.

LEY 18/2002, de 5 de julio, de Cooperativas de Cataluña.

LEY 20/1990, de Régimen fiscal de cooperativas.

LEY 22/2013, de 23 de diciembre, de presupuestos generales del Estado para el año 2014.

LEY 27/1999, de 16 de julio, de cooperativas.

LEY 85/98 de la Assembleia da República, de 16 de diciembre, Estatuto Fiscal Cooperativo.

LEY de Cantabria 6/2013, de 6 de noviembre, de Cooperativas de Cantabria.

LEY del Principado de Asturias 4/2010, de 29 de junio, de Cooperativas.

LEY FORAL 14/2006, de 11 de diciembre, de Cooperativas de Navarra. 
MARÍ VIDAL, S. y POLO-GARRIDO, F. (2012 a) Análisis de los efectos de la introducción de las normas sobre los aspectos contables de las sociedades cooperativas (Orden EHA/3360/2010). En II Jornada sobre emprendimiento social y colectivo, CEGEA, Valencia, España.

MARÍ VIDAL, S. y POLO-GARRIDO, F. (2012 b) Las recientes normas sobre los aspectos contables de las sociedades cooperativas (Orden EHA/3360/2010) desde la perspectiva de sus usuarios. En XIV Jornadas de Investigadores en Economía Social y Cooperativa, CIRIEC - España, San Sebastián, España.

MARÍN SÁNCHEZ, M.M.; MARÍ VIDAL, S.; SEGUÍ-MAS, E. y MATEOS-RONCO, A. (2012) Las pérdidas en la cooperativa y su tratamiento en función de la ubicación: evaluación contable y fiscal. En XIV Jornadas de Investigadores en Economía Social y Cooperativa, CIRIEC - España, San Sebastián, España.

MONTOLIO HERNÁNDEZ, J.M. (2000) Legislación cooperativa en la Unión Europea. Ministerio de Trabajo y asuntos sociales, Madrid, Instituto de Fomento de la Economía Social.

MÜNKNER, H.H. (2008) Taxation of co-operatives and in some EU Member States, European Research Institute, Trento, Münckner.

ORDEN EHA/3360/2010, de 21 de diciembre, por la que se aprueban las normas sobre los aspectos contables de las sociedades cooperativas.

PHILIPWOSKY R (1994) Taxation of co-operatives. In Dülfer, Eberhard. International Handbook of Co-operatives organisations, Göttingen.

REAL DECRETO LEGISLATIVO 4/2004, de 5 de marzo, por el que se aprueba el texto refundido de la Ley del Impuesto sobre Sociedades.

REAL DECRETO-LEY 4/2013, de 22 de febrero, de medidas de apoyo al emprendedor y de estímulo del crecimiento y de la creación de empleo.

RODRIGO RUIZ, M.A. (2010) Consideraciones sobre el régimen fiscal de las cooperativas. Problemas actuales y líneas de reforma. CIRIEC-España, Revista de Economía Pública, Social y Cooperativa, № 69 , pp. 9-26.

ROMERO CIVERA, A. (1992) Los cambios contables y fiscales dificultan la armonización con la legislación cooperativa. Agricultura y cooperación, $N^{o}$ 98, pp. 21-23.

ROMERO CIVERA, A. (2010) La fiscalidad aplicada a cooperativas en Europa y la reforma del régimen fiscal en España. CIRIEC-España, Revista de Economía Pública, Social y Cooperativa, № 69, pp. 91-118. 